\title{
Lignin-containing Adhesion Enhancer for Wood-plastic Composites
}

Galia Shulga,* Brigita Neiberte, Jevgenijs Jaunslavietis, Anrijs Verovkins, Sanita Vitolina, Vadims Shakels, and Sandra Livcha

A lignin-containing adhesion enhancer, a water-soluble polyelectrolyte complex (LPEC) composed of soda hardwood lignin (HASL) and polyethylenimine $(\mathrm{PEI})$, and the effect of the treatment of hydrolyzed and ammoxidized aspen sawdust with the LPEC nanoparticles were studied relative to the properties of wood-plastic composites (WPCs) based on recycled polypropylene (rPP). The presence of the excess of free amine groups and salt bonds between PEI and soda lignin, forming hydrophobic sites in the LPEC structure, caused the enhanced surface activity of the LPEC. The treatment with the LPEC nanoparticles increased the content of the fixed nitrogen in the modified sawdust samples and was accompanied by decreasing their water sorption and increasing contact angles that favored the decrease in the polar part of their surface free energy. The decreasing wetting ability enhanced the mechanical and water sorption properties of the obtained WPC samples. The improvement of the interfacial adhesion between the nitrogen-containing groups of the treated sawdust and the oxygen-containing groups of rPP was explained by the formation of both covalent and physicochemical bonds.

Keywords: Adhesion enhancer; Soda lignin; Hardwood sawdust; Polyelectrolyte complex nanoparticles; Recycled polypropylene; Wood-plastic composite

Contact information: Department of Lignin Chemistry, Latvian State Institute of Wood Chemistry, Riga, Latvia; *Corresponding author: galshulga@inbox.lv

\section{INTRODUCTION}

The principles of the Circular Economy (Korhonen et al. 2018) dictate the sustainable, rational, and efficient management of wastes and by-products because their annual volume remarkably increases and negatively affects the environment. In contrast, waste recovery can provide a huge secondary resource for producing new materials and reducing their costs. Great attention must be drawn to the rational and effective utilization of both wood and polymer wastes.

The use of wood fibers as a filler for obtaining wood-plastic composites (WPCs), due to their renewability, easy availability, low cost, biodegradability, light mass, enhanced filling degree, etc., is widely known (Faruka et al. 2012; Pickering et al. 2016). They are mainly used for building and construction applications, in the automobile industry, for production materials for domestic and technical needs, etc. According to the NOVAInstitute's statistical data, the demand for WPCs is growing globally (Carus et al. 2014).

Recycled polyolefins are among the major components of global municipal solid waste, and they present a promising raw material source for producing WPCs due to their large volume and low cost. The use of recycled synthetic polymers as a polymer matrix in 
WPCs is a promising practice for rational recovering of these wastes and reducing their load on the environment (Najafi 2013; Turku et al. 2018).

The high-performance properties of WPCs suffer from the problem of low compatibility between the hydrophobic polymer matrix and the hydrophilic wood filler. The improvement of the adhesion affinity between polyolefins and wood fibers in WPCs can be achieved by the modification of the fiber surface and/or the application of specific functional additives such as compatibilizers, coupling agents, or adhesion enhancers (Farsi 2012; Kabir et al. 2012). The functional additives can improve the properties of the filler particles, thus preventing their agglomeration and improving the adhesion interaction between a wood filler and a polymer matrix at the interface. They are applied in a small amount of 3 to $5 \mathrm{wt} \%$ by dry mass of the composite (De La Orden et al. 2010). The treatment of the wood surface with the functional additive can be done various ways, for example, by its addition as a solid ingredient in the raw blend of a polymer with a wood filler before their processing, by immersion or dipping of wood fibers in a solution, containing the functional additive, or by spraying this solution on the wood particle's surface before processing. The treatment of wood fibers by their immersion in the adhesion enhancer solution is an effective and inexpensive method for improving the properties of WPCs, allowing even distribution and adsorption of the enhancer over the wood surface before mixing with a polymer matrix. The choice of the adhesion enhancer is determined by its chemical composition, structure, molecular mass, reactivity, and depends on the origin of lignocellulosic fibers (wood species), fiber properties (specific surface area, porosity, surface charge density, etc.), filling degree, type of processing (extrusion, injection molding, pressing, etc.), and the required end-use properties of the WPCs (Lu et al. 2000; Kabir et al. 2012).

In the last decades, silanes, isocyanates, maleic anhydride-grafted polypropylene (PP-g-MAH), and polyethylene (PE-g-MAH) have been applied as interface adhesion modifiers for formulation of WPCs (La Mantia and Morreale 2011). The application of polyethylenimine (PEI) as a coupling agent for increasing the compatibility between a lignocellulosic filler and a polymer matrix is also known (De La Orden et al. 2007, 2010). Unfortunately, the most used functional additives for improving interfacial adhesion at the filler/polymer interface, such as maleated polyolefins, are synthetic, high-priced, and not biodegradable. In addition, maleated polyolefins can react with humidity during storage, with the release of free acid that can decrease their functional effectiveness (Taib et al. 2006). Many attempts have been made to use biodegradable polymers and their modified derivatives for obtaining the interface adhesion modifiers for application in WPCs, including lignin (La Mantia and Morreale 2011).

Lignin, a wood polymer, is the second most available polymer next to cellulose and comprises 25 to $30 \%$ of the non-fossil organic molecules on earth (Sarkanen and Ludwig 1971). The vast majority of technical lignins are co-products of pulp mills, where they are combusted in the form of lignin-rich black liquor streams to recycle process chemicals and produce steam for electrical energy generation. Lignin's great economic and industrial potential constitutes the real challenge of any study dedicated to valorization of lignins for their rational and effective utilization for obtaining value-added products (Karunarathna and Smith 2020). 
Due to the peculiarities of the chemical structure, lignin can be included in blends with thermoplastic polymers, performing the function of a compatabilizer. Attempts have been made for the development of methods for lignin modification by its complexation with metal ions, hydroxyalkylation, esterification, alkylation, grafting with synthetic polymers and oligomers, mixing with reactive synthetic compatibilizers, etc. (Hu et al. 2014). Each of these methods had drawbacks, such as the enhanced product cost, complicated technique, use of organic solvents, and high applied temperatures and pressures. Only a few lignin valorization products, mainly the etherified lignin products, met the requirements of an interfacial adhesion enhancer for WPCs (Mariotti et al. 2014; Kun and Pukanszky 2017).

Polyelectrolyte complexes (PECs) are polyelectrolytes self-assemblies that are formed because of the interaction of oppositely charged polyelectrolytes (Tsuchida 2008). The driving force of their formation is the gain in entropy caused by the release of lowmolecular-mass counter ions due to electrostatic interactions. Other types of interactions, such as hydrogen bonding and hydrophobic interactions, can additionally contribute to the complexation process. Due to the manifested adhesive and sorption properties, PECs have great potential and multiple practical applications in various practical fields, namely, in medicine as drug deliverers (Lankalapalli and Kolapali 2009; Verma and Verma 2013), for obtaining binders (Yuan et al. 2014), soil improving agents (Shulga et al. 2001, 2007), macrosurfactants (Shulga et al. 2009), etc. One of the prominent applications of PECs is the improvement properties of cellulose fibers and inter-fiber bonding (Gärdlund et al. 2003; Maximova et al. 2005; Hubbe 2006; Gärdlund et al. 2007; Hubbe et al. 2008; Ankerfors et al. 2009) in the papermaking process.

The polyanionic nature of soluble lignins enables it to interact with cationic polymers in aqueous media with the formation of lignin-containing polyelectrolyte complexes (LPECs) (Ström and Stenius 1981; Shulga et al. 1984; Li and Pelton 1992; Lappan et al. 1997). The LPECs can be fully soluble in water, form stable colloidal systems, or precipitate. The behavior depends on many factors, including the base strength of the cationic polymer, the interacting polyelectrolytes molecular masses, their charge densities, the lignin/polycation charge ratio, the medium's $\mathrm{pH}$, and the ionic strength. Water-soluble polyelectrolyte complexes at the low concentrations in dilute aqueous solutions may be regarded as specific block-copolymers, consisting of hydrophilic and hydrophobic blocks, forming "core-shell" nanoparticles (Kabanov 2005; Pergushov et al. 2010). The charge-compensated structural fragments of a polyelectrolyte complex contain charged polyelectrolytes that relate to each other by salt bonds and form a hydrophobic "core", while the disconnected regions of polyelectrolyte chains are in the "shell" of the nanoparticles. In contrast, the water-soluble LPECs may be regarded as polyampholytes, present in the polymer chains' positive and negative ionic groups. It is known that the polyampholyte structural features are the reason for their pronounced ability to be adsorbed on cellulosic surfaces, adapting to the changing external conditions at the interface (Hubbe 2006). The change in the charge ratio of the interacting polyelectrolytes, $\mathrm{pH}$ values, and ionic strength can dramatically affect their adsorption on the interfaces (Shulga et al. 2002; Maximova et al. 2005; Hubbe 2006).

The aim of this study was to obtain an enhancer of interfacial adhesion in the form of a water-soluble polyelectrolyte complex, composed of soda lignin synthetic cationic polymer, and to study its effect on the properties of a wood-plastic composite based on recycled polypropylene. 


\section{EXPERIMENTAL}

\section{Materials}

Aspen sawdust, representative of a waste of the mechanical processing of aspen wood (Populus tremula), was supplied by a Latvian company (4 PLUS Ltd., Aizkraukle, Latvia). The sawdust was characterized by elemental analysis (Elementar Analysensysteme, Langenselbold, Germany) and wood polymers' composition according to the procedures of Klason and Kürschner for lignin as per the TAPPI T222 om-11 standard (2011) and for cellulose as per the TAPPI T203 cm-99 standard (1999), respectively. The hemicellulose content was determined according to the TAPPI T264 om97 (1997) standard. The elemental composition of the aspen wood sawdust was the following: $48.77 \% \mathrm{C}, 6.25 \% \mathrm{H}, 44.77 \% \mathrm{O}, 0.11 \% \mathrm{~N}$, and $0.10 \% \mathrm{~S}$. The contents of cellulose, lignin, hemicellulose, extractives, and ash in the aspen wood were $50.6 \%, 18.5 \%$, $28.7 \%, 4.51 \%$, and $0.4 \%$, respectively. The extractives and ash content were determined according to Zakis (1994).

Soda lignin, as a component of the LPEC, was obtained by the delignification of the acid hydrolyzed aspen sawdust in a laboratory reactor under the following laboratory conditions: hydromodulus $-1 / 7, \mathrm{NaOH}$ concentration $-4.0 \%$, temperature $-165^{\circ} \mathrm{C}$, and duration $-1.5 \mathrm{~h}$. The hydrolyzed aspen soda lignin (HASL) was separated from the cooking solution of aspen sawdust by precipitation with $20 \%$ sulfuric acid, with the following: filtration, washing with hot distilled water, and subsequent drying. The content of Klason lignin in the sample was $90.8 \%$ as per the TAPPI T222 om-02 standard (2002). The chemical composition of the lignin was determined by elemental analysis (Elementar Analysensysteme $\mathrm{GmbH}$, Langenselbold, Germany). Functional group analysis was performed using the Fibok-Shvappakh method for methoxyl groups, the acetylation method for aliphatic hydroxyl groups, the interaction with hydroxylamine hydrochloride for carbonyl groups, and potentiometric and conductometric titration for phenolic hydroxyl and carboxyl groups, according to Zakis (1994). The purified lignin had the following chemical composition: $62.3 \% \mathrm{C}, 6.5 \% \mathrm{H}, 0.15 \% \mathrm{~N}, 0.25 \% \mathrm{~S}, 30.8 \% \mathrm{O}, \mathrm{OCH} 3-19.3 \%$, total $\mathrm{OH}-5.6 \%$, phenolic $\mathrm{OH}-3.5 \%$, aliphatic $\mathrm{OH}-2.1 \%, \mathrm{COOH}-2.1 \%$, and $\mathrm{CO}-$ $3.9 \%$. The average molecular weights of the lignin $\left(M_{\mathrm{w}}, M_{\mathrm{n}}\right)$, determined with a SECMALS20 (Malvern Panalytical, Malvern, United Kingdom) in dimethyl sulfoxide (DMSO) with lithium bromide as an eluent at $60^{\circ} \mathrm{C}$, were close to $10.5 \mathrm{kDa}$ and $5.7 \mathrm{kDa}$, respectively.

High molecular branched polyethylenimine (PEI, $\left.M_{\mathrm{w}}=750 \mathrm{kDa}, M_{\mathrm{n}}=60 \mathrm{kDa}\right)$ in a 50\% water solution, supplied by Acrōs Organics (ThermoFisherScientific, Fair Lawn, NJ, USA), was used as a component of the LPEC.

The used reagents were purchased from Sigma-Aldrich Chemie (Taufkirchen, Germany).

\section{Activation of aspen sawdust}

The hydrolyzed sawdust was obtained by low temperature hydrolysis with the dilute solution of hydrochloric acid under mild conditions $\left(60{ }^{\circ} \mathrm{C}, 5 \mathrm{~h}\right.$, and weight ratio of wood particles to water 1:20) according to Shulga et al. (2016b). The ammoxidation of the hydrolyzed sawdust particles was completed by adding aspen microparticles to a $\mathrm{NH}_{4} \mathrm{OH}$ solution in the presence of persulfate ammonium as an oxidizing agent according to Shulga et al. (2016c). The content of the fixed organic nitrogen in the ammoxidized sawdust was equal to 1.09. Both the hydrolyzed and ammoxidized sawdust particles were washed with 
double-distilled water using a Büchner funnel and a Bunsen flask, then dried to a neutral medium, dried, and milled with a planetary ball mill (Retsch, Haan, Germany) for 15 min at $300 \mathrm{rpm}$. After sieving, a fraction of the particles less than $100 \mu \mathrm{m}$ was used for the treatment with the LPEC. The sieving was performed with a Vibratory micromill "Pulverisette 0" (Frisch GmbH, Idar-Oberstein, Germany).

\section{Obtaining the LPEC}

An adhesion enhancer in the form of LPEC was obtained by adding $0.2 \%$ aqueous solutions of PEI to $0.2 \%$ alkaline aqueous solutions of HASL (1:1 on dry matter) at pH 10.5 and room temperature by stirring the reaction mixture at $200 \mathrm{rpm}$ using a magnetic stirrer (IKA RCT basic IKAMAG, IKA-Werke GmbH, Staufen, Germany) for 15 min. The soda lignin/PEI reaction mixture was prepared immediately before its application. Doubledistilled water was used for preparation of the solutions of HASL, PEI, and their reaction mixture.

Treatment of the sawdust microparticles with the LPEC

The treatment of the hydrolyzed and ammoxidized aspen particles, $<100 \mu \mathrm{m}$, was performed by immersion of $7.5 \mathrm{~g}$ of the modified aspen particles in $1 \mathrm{~L}$ of the prepared HASL/PEI reaction mixture for $24 \mathrm{~h}$ under constant slow stirring at room temperature. After the treatment, both the hydrolyzed and ammoxidized particles were washed to neutral $\mathrm{pH}$ and dried at $60{ }^{\circ} \mathrm{C}$ for $48 \mathrm{~h}$ and $2 \mathrm{~h}$ at $105^{\circ} \mathrm{C}$, respectively. The moisture content in the treated sawdust particles was less than $1.0 \%$. The content of nitrogen $(\mathrm{N})$ in the treated hydrolyzed sawdust was equal to $1.12 \%$, but in the treated ammoxidized particles it was $2.38 \%$.

\section{Preparation of WPC samples}

Recycled polypropylene (rPP) $\left(0.9 \mathrm{t} / \mathrm{m}^{3}, 5.2 \mathrm{~g} / 10 \mathrm{~min}\right.$ at $230{ }^{\circ} \mathrm{C}$, and $\left.2.16 \mathrm{~kg}\right)$ was used as a thermoplastic polymer matrix and supplied from a Latvian polymer recycling plant (Nordic Plast Ltd., Olaine, Latvia).

Before obtaining the WPC samples, the powdered rPP and aspen sawdust microparticles were mixed for $5 \mathrm{~min}$ at room temperature with a vibratory micromill PULVERISETTE 0 (Frisch GmbH, Idar-Oberstein, Germany). The samples for tensile and bending tests were prepared by the extrusion and moulding method using HAAKE MiniLab II and MiniJet II (Thermo Fisher Scientific, Karlsruhe, Germany) at a temperature of $175{ }^{\circ} \mathrm{C}$, a circulation time in the two-screw extruder of $5 \mathrm{~min}$, and a molding pressure of $60 \mathrm{MPa}$ at a temperature of $120{ }^{\circ} \mathrm{C}$. The content of the hydrolysed and ammoxidized sawdust microparticles in the WPC samples was $50 \mathrm{wt} \%$.

\section{Methods}

Surface tension

The surface tension $(\gamma)$ at the water-air interface for HASL and PEI aqueous solutions, as well as their reaction mixture, was measured by a Wilhelmy plate (Della Volpe and Siboni 2018), using a tensiometer K100M (KRUSS, Hamburg, Germany) at 25 ${ }^{\circ} \mathrm{C}$ with a circulation thermostat. The preparation of the samples and the measuring procedure were similar to those described by Shulga et al. (2006). Three replicates were made for each sample. 


\section{Zeta potential and size}

Zeta potential $(\zeta$ ) for the sawdust microparticles was measured with a dynamic light scattering device Zetasizer Nano SZ (Malvern Panalytical, Malvern, UK) at $25{ }^{\circ} \mathrm{C}$, using $0.1 \%$ suspensions of the particles in bi-distilled water. For circulation of the suspension, an autotitrator MPT-2 (Malvern Panalytical, Malvern, UK) was used. Three replicates were made for each modified sawdust.

\section{Viscometry}

Specific viscosity of soda lignin, PEI, and LPEC was determined using a capillary viscometer Ubbelohde (Schortt Instruments, Mainz, Germany) with the flow time of bidistilled water at $300 \pm 0.01 \mathrm{~s}$ at $25.0 \pm 0.1^{\circ} \mathrm{C}$, as described previously (Shulga et al. 2006). Three replicates were made for each sample.

\section{Potentiometric and conductometric titration}

The titration curves of HASL and PEI aqueous solutions were obtained using an InoLab Level 3 Multiparameter Meter (Wissenschaftlich-Technische Werkstätten, Weilheim, Germany) at constant stirring at $25^{\circ} \mathrm{C}$. For the adjustment of $\mathrm{pH}$ values, $0.1 \mathrm{M}$ $\mathrm{NaOH}$ and $0.1 \mathrm{M} \mathrm{HCl}$ solution were used.

\section{UV spectroscopy}

The UV-spectra were recorded with a Genesys 10UVUV/VIS spectrophotometer (Termo Fisher Scientific, San Jose, CA, USA).

\section{Wetting properties of WPC samples}

To study the surface properties, WPC samples with dimensions of $60 \times 10 \times 1 \mathrm{~mm}^{3}$ were prepared. Before testing, the samples were conditioned at $60{ }^{\circ} \mathrm{C}$ in a drying cabinet for $24 \mathrm{~h}$ and then stored for $1 \mathrm{~h}$ at room temperature in a desiccator with phosphorus pentoxide.

The advancing contact angles of the modified aspen particles and the WPC samples were measured with a tensiometer Kruss K100M (Kruss, Hamburg, Germany) using the Washburn (Washburn 1921) and Wilhelmy (Della Volpe and Siboni 2018) methods, respectively. The work of adhesion was calculated using the Young-Dupre equation (Eq. $1)$,

$$
W=\gamma(1+\cos \theta)
$$

where $W(\mathrm{mN} / \mathrm{m})$ is the work of adhesion, $\gamma$ is the liquid surface tension $(\mathrm{mN} / \mathrm{m})$, and $\theta\left(^{\circ}\right)$ is the contact angle.

The total surface free energy (SFE) and its dispersive (Lifshitz - Van der Waals interactions) and polar (Lewis acid-base interactions) components were calculated using the Owens-Wendt-Rabel-Kaelble method (Adamson and Gast 1997), for which the contact angle values with different liquids: water, DMSO, and diiodomethane were measured. For the calculation of SFE, a special software program (Advance Software Assurance, version 3.2, Kruss, Hamburg, Germany) provided in the tensiometer system was applied. Three replicates were tested for each sample and their arithmetic mean value was calculated.

The water vapor sorption of the composite samples was determined using the desiccator's method at a relative humidity of $98 \%$ and room temperature. Three replicates were made for each modified sawdust and WPC sample. 


\section{Mechanical tests}

Mechanical properties were determined using a universal machine "Zwick" (Zwick/Roell, Ulm, Germany) with a load capacity of $0.5 \mathrm{kN}$ at a rate of $50 \mathrm{~mm} / \mathrm{min}$ and $2 \mathrm{~mm} / \mathrm{min}$ for tensile and bending tests according to ASTM D638 (2007) and ISO 178 (2010) with the help of the software program (TestXpert I, Zwick/Roell, Ulm, Germany). Before testing, the samples were conditioned at $60{ }^{\circ} \mathrm{C}$ for $24 \mathrm{~h}$ and then placed in a desiccator with phosphorus pentoxide. Five replicates were made for each mechanical testing, and the standard deviation for each index was found.

Scanning electron microscopy (SEM)

The morphology of the obtained WPC samples was examined using a scanning electron microscope (Tesla, Brno, Czech Republic).

\section{Milling}

The milling of wood sawdust was carried out with a planetary ball mill (Retsch, Haan, Germany) and sieved using a mill "Pulverisette 0" (Frisch GmbH, Idar-Oberstein, Germany).

\section{RESULTS AND DISCUSSION}

\section{Adhesion Enhancer}

The working hypothesis of the study was the following: a water-soluble nonstoichiometric polyelectrolyte complex lignin/polyethyleneimine could ensure optimal physicochemical interaction with both a weakly negative surface of the rPP matrix and a negatively charged activated wood surface that would positively affect the compatibility between the wood filler and recycled polypropylene. A water-soluble, non-stoichiometric polyelectrolyte complex was obtained by the interaction of HASL and PEI in the reaction mixture with their mass ratio close to 1 . The content of amine groups in the cationic polymer was more than 3-fold higher than the content of phenolic hydroxyl and carboxyl groups in the soda lignin in the reaction mixture. The excess of the amine groups of PEI in the LPEC was motivated by their expenditure on the formation of covalent bonds with both the surface's carbonyl and carboxyl groups of sawdust, and the oxidized groups of the rPP during the processing.

The needed functional groups ratio in the reaction mixture HASL/PEI was chosen based on the results of potentiometric and conductometric titration of amine groups of the PEI (Fig. 1A) and phenolic hydroxyl and carboxyl groups of soda lignin (Fig. 1B). The initial $\mathrm{pH}$ value of the LPEC HASL/PEI water solution was alkaline ( $\mathrm{pH}$ 10.5). The alkaline medium of the LPEC HASL/PEI water solution was conditioned by the nucleophilic mechanism of the interaction between primary and secondary amine groups of the PEI and carbonyl and carboxyl groups of the modified sawdust and rPP during the processing, leading to the formation of the covalent bonds, namely, amide and imine (De La Orden et al. 2007, 2010), with both the wood filler and the recycled polymer matrix.

Table 1 shows the values of surface tension and specific viscosity of alkaline aqueous solutions of the LPEC HASL/PEI and its initial components - HASL and PEI, with the same concentrations, as well as the sizes and Zeta potential values of the LPEC, HASL, and PEI. 

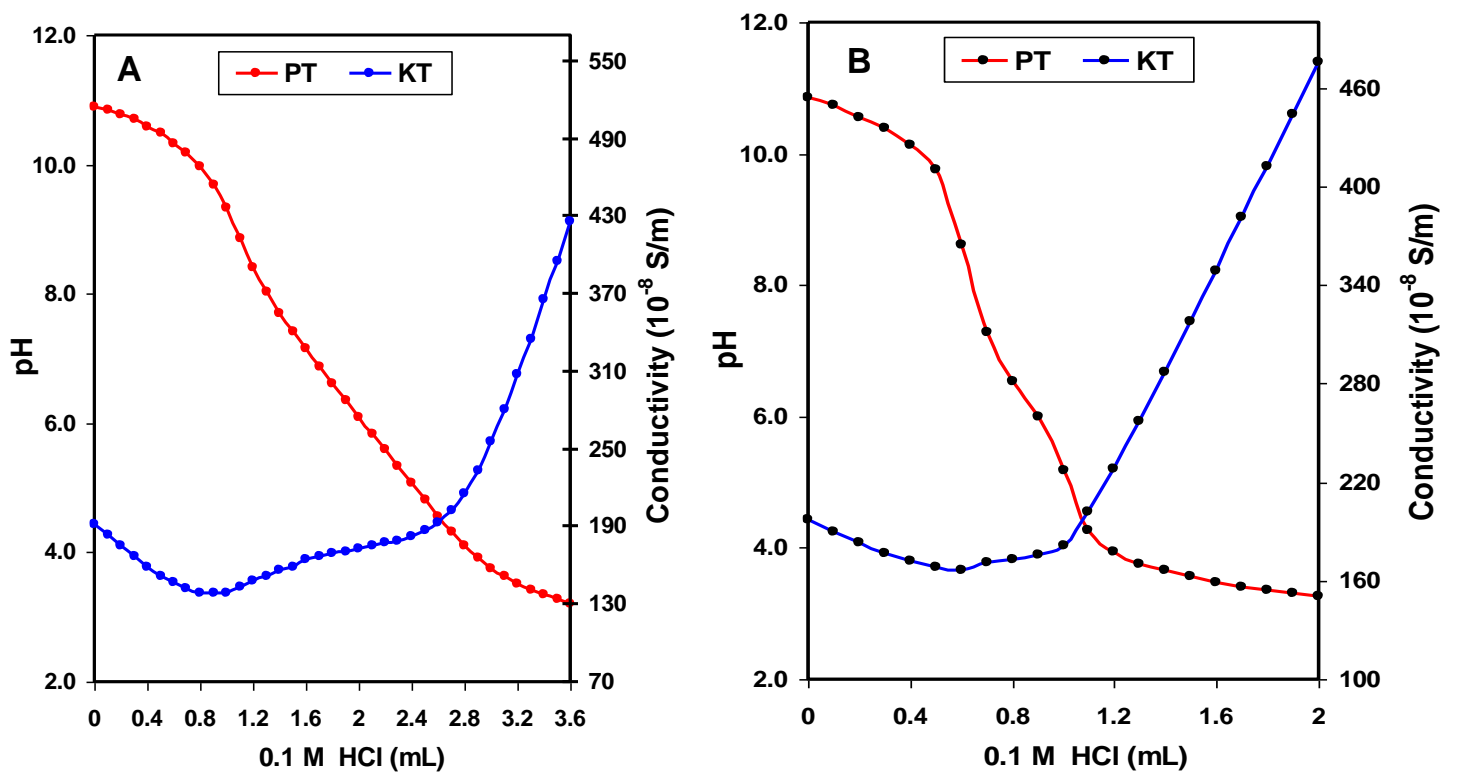

Fig. 1. Potentiometric (PT) and conductometric (KT) titration curves of $(A) 0.01 \% P E I$ and $(B)$ $0.01 \%$ HASL aqueous solutions

Table 1. Characteristics of $0.1 \% \mathrm{HASL}, \mathrm{PEI}$, and Aqueous Solutions of Polyelectrolyte Complex HASL/PEI (1:1) at pH 10.5

\begin{tabular}{|c|c|c|c|}
\hline Index & HASL & PEI & HASL/PEI \\
\hline Surface tension $(\mathrm{mN} / \mathrm{m})$ & 60.6 & 69.8 & 50.6 \\
\hline Zeta potential $(\mathrm{mV})$ & -23.1 & -7.5 & -19.0 \\
\hline Size $(\mathrm{nm})$ & $\mathbf{1 3 4}$ & $\mathbf{9 5}$ & $\mathbf{9 8}$ \\
\hline Specific viscosity & 0.019 & 0.045 & 0.056 \\
\hline
\end{tabular}

According to the data obtained, the LPEC was characterized by better surfaceactive properties than the initial components. Thus, the LPEC expressed lower surface tension at the air-water interface compared to the initial components. Simultaneously, the value of its specific viscosity in the alkaline solution was less than the additive value of specific viscosity of the initial components, which may indicate a compact structure of the formed LPEC nanoparticles. The sizes of the nanoparticles of HASL, PEI, and the LPEC were in the range of 108 to $134 \mathrm{~nm}$. The diameter of the formed LPEC nanoparticles was smaller than the size of the lignin nanoparticles, and close to that of the PEI nanoparticles. Taking into account the fact that the amino groups of PEI at this $\mathrm{pH}$ were practically not protonated, it can be assumed that, under these conditions, the LPEC nanoparticles can be only formed by H-bonding between the non-protonated amino groups of PEI, carbonyl ( $\alpha$ and $\beta$-ketogroups, aldehyde groups, quinone groups), and hydroxyl groups of soda lignin. At the same time, the visible increase in the intensity of the absorption curve of the UV spectrum of the alkaline solution of the LPEC HASL/PEI relative to the lignin solution (PEI does not absorb in the UV region) (Fig. 2) most pronounced in the range of 260 to $340 \mathrm{~nm}$, testified the presence of the electrostatic interaction in the LPEC between the induced protonated amine groups of PEI and phenylpropane chains of soda lignin, containing non-conjugated phenol hydroxyl groups (absorption in the range of 280 to 300 $\mathrm{nm}$ ), phenol hydroxyl groups conjugated with $\alpha$-carbonyl groups (absorption at $310 \mathrm{~nm}$ ), and carbonyl and carboxyl groups (absorption at 328 and $345 \mathrm{~nm}$ ) (Faix 1992). 


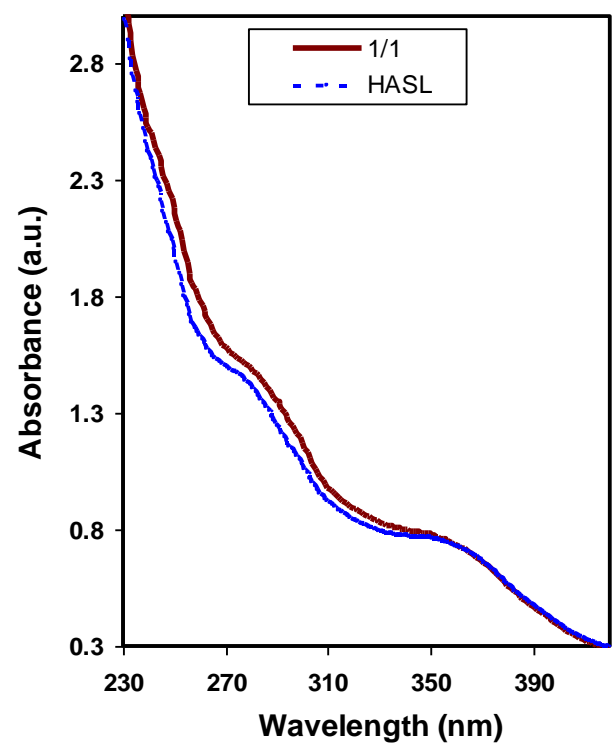

Fig. 2. UV spectra of dilute alkaline solutions of HASL and LPEC (1:1)

The change in the absolute value of $\zeta$ potential of the LPEC nanoparticles by 4.1 $\mathrm{mV}$ relative to the $\zeta$ potential of the negatively charged soda lignin (Table 1 ) could also confirm the presence of the electrostatic interaction between HASL and PEI at the alkaline $\mathrm{pH}$ value. The salt bonds between the positively charged amine groups of PEI and negatively charged functional groups of soda lignin are electrically neutral and can form hydrophobic sites in the resulting LPEC structure. Thus, the formation of the water-soluble LPEC in an alkaline medium may be regarded as a result of hydrogen and electrostatic interactions between the functional groups of soda lignin and PEI. It is obvious that hydrophobic, $\pi$-cationic and weak Van der Waal's interactions can also contribute to the formation of the LPEC nanoparticles. The presence of the excess of the free non-protonated amine groups, capable of forming imine and amide bonds with carbonyl and carboxyl groups, as well as the presence of salt bonds, forming hydrophobic sites in the LPEC, suggested an enhanced reactivity of the obtained LPEC HASL/PEI nanoparticles relative to the wood microparticles and the oxidized surface of the rPP.

\section{Sawdust Filler}

Table 2 shows that both the mild acid hydrolysis and ammoxidation affected the chemical composition of the aspen sawdust. The cleavage of ester and ether linkages in the lignocellulosic matrix of the wood during both modifications was accompanied by decreasing the content of hemicelluloses and lignin, but by increasing the relative content of cellulose. This chemical change led to the chemical activation of the lignocellulosic matrix surface and facilitated its accessibility for the following treatment with the adhesion enhancer. 
Table 2. Aspen Sawdust Chemical Composition Depending on the Modification

\begin{tabular}{|c|c|c|c|c|c|}
\hline Treatment & $\begin{array}{c}\text { Cellulose } \\
(\%)\end{array}$ & Lignin (\%) & $\begin{array}{c}\text { Hemicellulose } \\
(\%)\end{array}$ & $\mathbf{N}(\%)$ & O/C \\
\hline Initial & 50.6 & 18.5 & 28.7 & 0.11 & 0.26 \\
\hline Hydrolysis & 52.6 & 18.2 & 24.5 & 0.17 & 0.24 \\
\hline Ammoxidation & 54.8 & 17.5 & 22.4 & 1.09 & 0.25 \\
\hline
\end{tabular}

The treatment of the modified samples - hydrolyzed and ammoxidized microparticles - with the LPEC was performed via their immersion in an alkaline aqueous solution at a room temperature. As a result, the content of the fixed nitrogen in the hydrolyzed and ammoxidized sawdust after drying increased from 0.17 to $1.15 \%$ and from 1.09 to $2.38 \%$, respectively (Table 3 ). Because the sawdust particles modified with the hydrolysis and ammoxidation had a negative charge in the alkaline medium, due to the dissociation of the surface phenolic hydroxyl and carboxyl groups, it can be supposed that the adsorption of the LPEC nanoparticles on the negatively charged wood surface proceeded due to physicochemical interactions including Van der Waals' forces, hydrophobic interactions, and the formation of acceptor-donor complexes between the functional groups. Such type of the interaction of the LPEC nanoparticles with the modified wood surface may resemble the adsorption of the negatively charged lignin particles onto the negatively charged pulp fibers (Wågberg and Annergren 1997).

The main drawback of wood particles as a WPC filler was their pronounced hydrophilicity, which was expressed in the enhanced wetting ability. According to Table 3 , the more pronounced ability to take up water vapor belonged to the initial milled sawdust. The content of water in these particles during $25 \mathrm{~d}$ reached almost $18 \%$ (on dry matter). This was caused by the presence of the hydroxyl and carboxyl groups emerging because of the mechanical treatment of the sawdust. This was evidenced by the highest negative value of the $\zeta$ potential for the milled particles, which correlates with the amount of the charged groups present at the sawdust surface.

Table 3. Characteristics of Initial, Modified, and Treated Modified Aspen Sawdust Microparticles $(<100 \mu \mathrm{m})$

\begin{tabular}{|c|c|c|c|c|c|}
\hline Sample & $(-) \zeta(\mathbf{m V})$ & $\begin{array}{c}\text { Contact } \\
\text { Angle } \\
(\mathbf{(})\end{array}$ & $\begin{array}{c}\text { Adhesion Work } \\
(\mathbf{m N} / \mathbf{m})\end{array}$ & $\begin{array}{c}\text { Water } \\
\text { Sorption } \\
(\%)\end{array}$ & $\begin{array}{c}\text { Fixed } \\
\text { Nitrogen } \\
(\%)\end{array}$ \\
\hline Initial & 18.2 & 79.70 & 85.82 & 17.9 & 0.11 \\
\hline Hydrolysed & 16.5 & 85.10 & 79.02 & 15.8 & 0.17 \\
\hline $\begin{array}{c}\text { Hydrolysed }+ \\
\text { LPEC }\end{array}$ & 15.8 & 87.20 & 75.30 & 14.9 & 1.13 \\
\hline Amooxidised & 16.3 & 84.30 & 80.03 & 16.4 & 1.09 \\
\hline $\begin{array}{c}\text { Amooxidised }+ \\
\text { LPEC }\end{array}$ & 15.3 & 88.00 & 74.90 & 14.5 & 2.38 \\
\hline
\end{tabular}

The modified sawdust microparticles, namely hydrolyzed and ammoxidized ones, adsorbed water to a lesser extent, $15.8 \%$ and $16.4 \%$, respectively, and had lower negative values of the $\zeta$ potential (Table 3). Evidently, the lower ability to uptake water may be conditioned by the reduction of the content of hemicelluloses by 4 to $6 \%$ (Table 1 ) in both samples, as well as the formation of covalent amide bonds in the ammoxidized sample. The treatment of the modified sawdust particles with the LPEC led to a further increase in 
their surface hydrophobicity, which was reflected in a decrease in the amount of adsorbed water by 6 to $12 \%$ relative to the untreated modified samples and 20 to $23 \%$ relative to the initial sawdust. Evidently, the decreasing ability to be wetted by water was a result of the formation of imine and amide bonds between the free amino groups of the LPEC and the carbonyl and carboxyl groups of the lignocellulosic matrix of the modified sawdust (Shulga et al. 1982; De La Orden et al. 2007).

According to Table 3, with decreasing of the absolute values of the $\zeta$ potential, the contact angles of the treated sawdust particles were increased. Partial removal of hemicelluloses in the hydrolyzed sawdust sample and the formation of amide bonds in the ammoxidized sawdust sample increased their contact angles from $79.7^{\circ}$ (for the initial particles) to $85.1^{\circ}$ (for the ammoxidized sample) and $84.3^{\circ}$ (for the hydrolyzed d sample). The lower contact angle of the ammoxidized sawdust particles, in comparison with the case of the hydrolyzed sawdust sample, may be caused by both the insufficient amount of the formed amide bonds due to the low content of the fixed nitrogen, and the presence of the hydrophilic carboxylic groups formed during the ammoxidation (Shulga et al. 2016c). The increase in the content of nitrogen in the ammoxidized particles treated with the LPEC nanoparticles allowed for an increase in their contact angle compared with the contact angle of the treated hydrolyzed sawdust particles. According to the values of the calculated adhesion energy (Table 3), the treated modified sawdust particles had lower affinity to water relative to the initial and untreated modified sawdust samples.

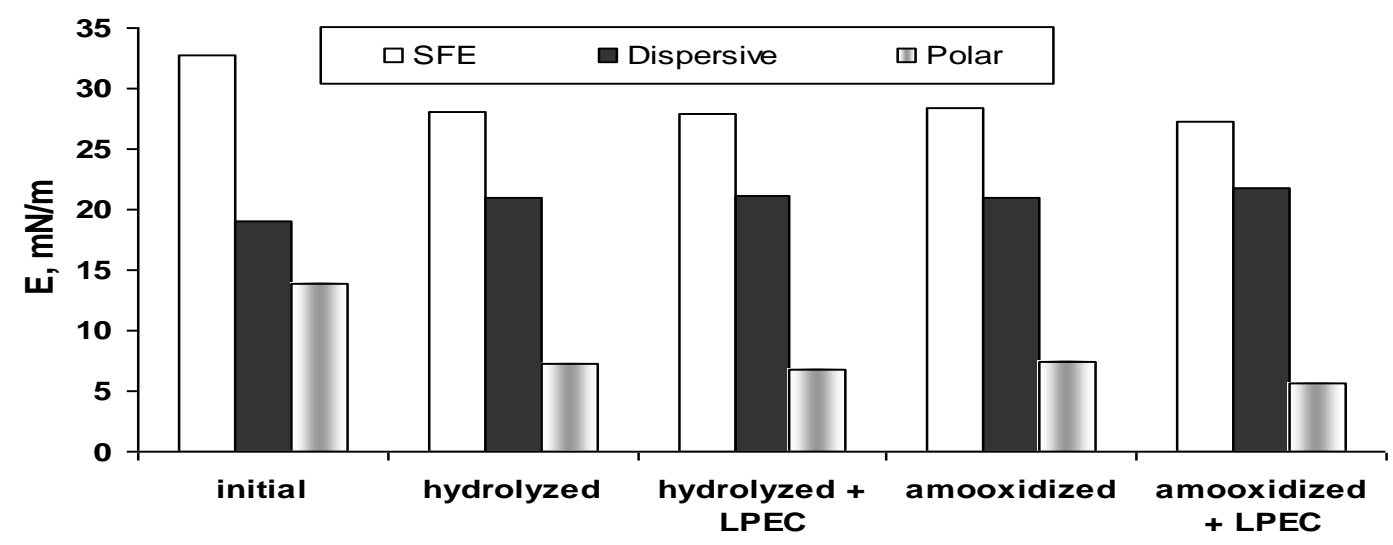

Fig. 3. Surface free energy, its polar and dispersive parts of the sawdust samples

The SFE, as well as its polar and dispersive parts for sawdust samples, was calculated using different organic liquids (Fig. 3). It was found that the SFE of the initial sawdust was higher than that of all the modified sawdust samples. At the same time, the treatment with the LPEC nanoparticles, to a greater extent, affected the polar part of the free energy of the sawdust samples.

The treatment with the LPEC reduced the polar part of the free energy of the ammoxidized sample by almost $57 \%$, while the decrease in the polar part of the SFE of the treated hydrolyzed sawdust reached $52 \%$ relative to the untreated sawdust. The observed drop of the polar part of the SFE of the treated modified samples, compared with the untreated modified ones, could be explained by the formation of new amide and imine bonds between carboxyl and carbonyl groups, being on the surface of the modified sawdust 
samples, and the free amine groups of PEI contained in the LPEC nanoparticles. The dispersion force component of the treated modified sawdust samples also increased, compared with the initial and untreated modified sawdust, although these changes were less pronounced.

\section{Wood-plastic Composites}

The composite samples were prepared from rPP powder and the aspen sawdust microparticles according to the following scheme (Fig. 4).

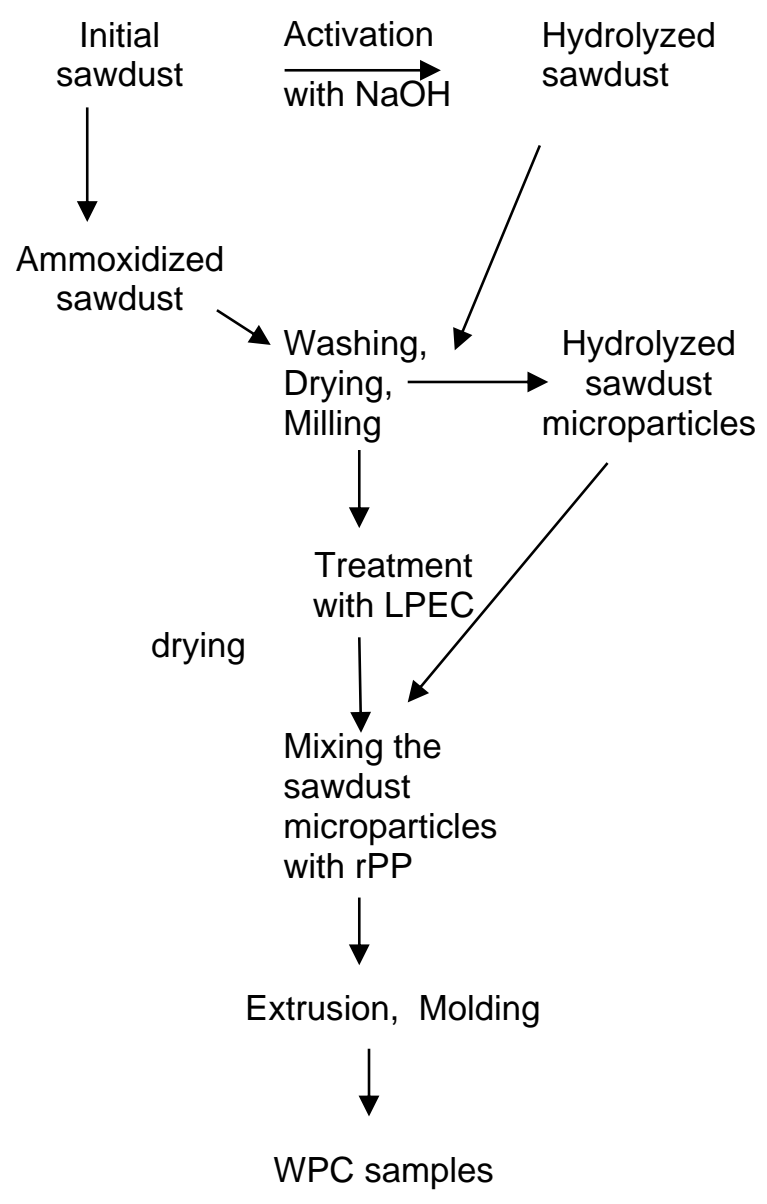

Fig. 4. Scheme of preparing the WPC samples

The values of contact angles, adhesion energy, and water sorption after $25 \mathrm{~d}$ for the obtained wood-polymer composites are shown in Table 4.

Table 4. Characteristics of Wetting Properties of the WPC Samples

\begin{tabular}{|c|c|c|c|}
\hline WPC Sample & CA $\left(^{\circ}\right)$ & $\begin{array}{c}\text { Adhesion Work } \\
(\mathbf{m N} / \mathbf{m})\end{array}$ & $\begin{array}{c}\text { Water Sorption } \\
(\mathbf{w t} \%)\end{array}$ \\
\hline Initial/milled & 90.6 & 70.8 & 3.4 \\
\hline Hydrolyzed + LPEC & 96.3 & 64.4 & 2.5 \\
\hline $\begin{array}{c}\text { Ammooxidized }+ \\
\text { LPEC }\end{array}$ & 97.8 & 62.7 & 2.3 \\
\hline
\end{tabular}


The comparison of the values given in Tables 3 and 4 shows that the composite samples had larger contact angles, lower adhesion energy, and remarkably lower water sorption compared with the modified sawdust particles, which was gained by the hydrophobicity of the polymer matrix in the WPC samples.

According to Table 4, the WPC samples filled with the treated hydrolyzed and ammoxidized microparticles were characterized by an increase in the contact angle values from $90.6^{\circ}$ to $96.3^{\circ}$ and $97.8^{\circ}$, a decrease in the adhesion energy from 70.8 to $63.4 \mathrm{mN} / \mathrm{m}$ and $62.7 \mathrm{mN} / \mathrm{m}$, and a reduction in the water sorption during 25 days from $3.4 \%$ to $2.5 \%$ and $2.3 \%$, respectively, compared with the WPC sample containing the initial milled sawdust. These results indicated that the improvement of the compatibility between the filler and the recycled polymer matrix during the processing could be explained by their physicochemical interaction at the interface due to both the ion-pairing within a polyelectrolyte complex system, the formation of covalent bonds (amide, imine) between the oxidized groups (carbonyl, carboxyl) of rPP, and the free uncharged amino groups of the LPEC nanoparticles located on the treated modified wood surfaces, and H-bonding between the nitrogen-containing groups of the filler and the oxygen-containing groups of rPP.

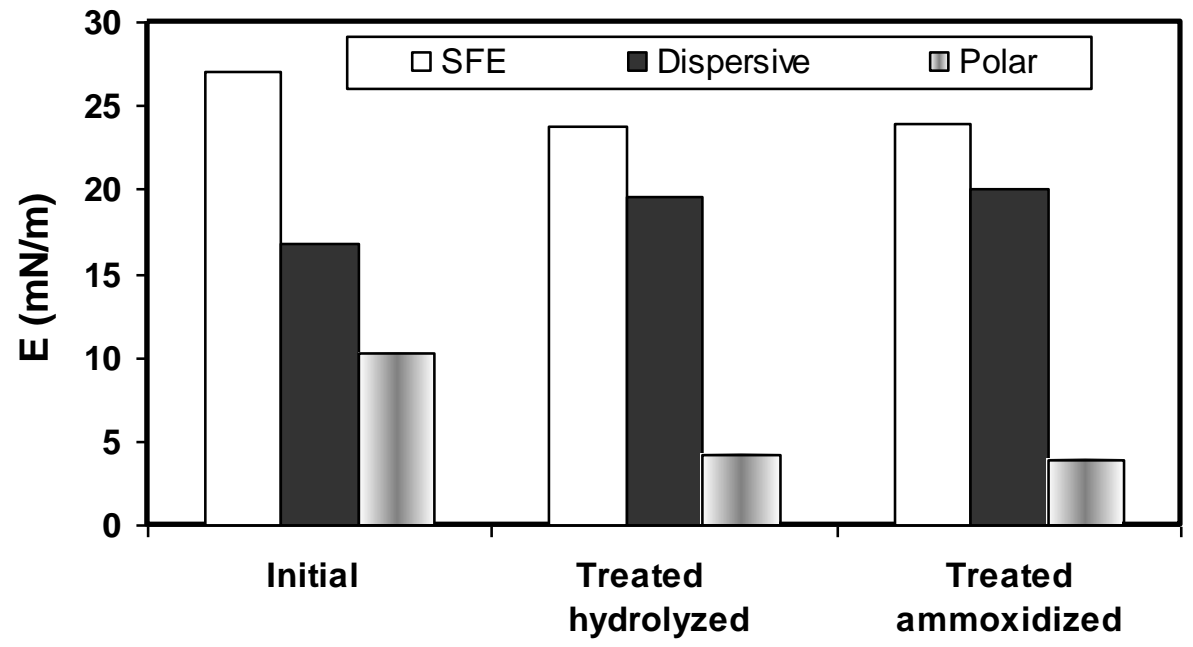

Fig. 5. Surface free energy, its polar and dispersive parts of the WPC samples

The negligible difference in the values of the indices (Table 4) for the WPC samples filled with the treated hydrolyzed and ammoxidized sawdust particles may be caused by the insufficient difference in their hydrophobicity.

According to Fig. 5, the SFE of the WPC samples filled with the initial sawdust was 12.9 to $13.3 \%$ higher than the same values for the WPC samples, containing the treated modified sawdust microparticles. The decrease in the SFE values for the WPC samples, containing the treated hydrolyzed and ammoxidized particles, was gained by an essential drop in their SFE polar part by almost 2.4 and 2.6 times, respectively, compared to the WPC sample filled with the initial sawdust. This could be explained by the decrease in the hydrophilicity of the modified sawdust particles treated with the LPEC. Simultaneously, there was a decrease in the dispersion force component of the WPC samples, which was less pronounced than the drop in their SFE polar part. The remarkable decrease in the polar part of SFE and the increase in the dispersion force component of SFE for the WPC 
samples, containing the modified particles treated with the LPEC, testified to the effectiveness of the application of the LPEC nanoparticles as an adhesion enhancer, which was able to improve the interfacial adhesion by forming physicochemical bonds with both the modified sawdust particles and the recycled polymer matrix. The insignificant difference in the values of free energy and its polar and dispersion parts for the WPC samples filled with the hydrolyzed and ammoxidized sawdust microparticles, treated with LPEC, was reflected in the insignificant difference in their hydrophobicity (Table 3), and can be explained by the minor difference in the hydrophobicity of the used modified sawdust particles.

The formation of covalent and hydrogen bonds between the filler and recycled polypropylene led to the structuring of the interfacial layers and the reinforcement of the interfacial adhesion in the composites. These factors are reflected in the improvement of the mechanical properties of the obtained wood-polymer composite samples (Table 5).

Table 5. Mechanical Properties of the WPC Samples

\begin{tabular}{|c|c|c|c|c|c|c|}
\hline Filler & $\begin{array}{c}\text { Tensile } \\
\text { Strength } \\
(\mathbf{M P a})\end{array}$ & $\begin{array}{c}\text { Tensile } \\
\text { Modulus } \\
(\mathbf{M P a})\end{array}$ & $\begin{array}{c}\boldsymbol{\varepsilon}_{\mathbf{T}}^{*} \\
(\%)\end{array}$ & $\begin{array}{c}\text { Bending } \\
\text { Strength } \\
(\mathbf{M P a})\end{array}$ & $\begin{array}{c}\text { Bending } \\
\text { Modulus } \\
(\mathbf{M P a})\end{array}$ & $\boldsymbol{\varepsilon}_{\mathbf{B}}{ }^{* *}(\mathbf{m m})$ \\
\hline Initial/Milled & 26.2 & 725 & 11.5 & 29.1 & 1635 & 5.9 \\
& \pm 0.2 & \pm 12 & \pm 0.5 & \pm 0.2 & \pm 31 & \pm 0.2 \\
\hline Hydrolyzed + & 38.5 & 1028 & 5.1 & 41.5 & 3455 & 1.8 \\
LPEC & \pm 0.3 & \pm 15 & \pm 0.6 & \pm 0.4 & \pm 23 & \pm 0.3 \\
\hline Ammoxidized + & 39.8 & 1120 & 4.9 & 42.7 & 3510 & 1.6 \\
LPEC & \pm 0.4 & \pm 17 & \pm 0.8 & \pm 0.4 & \pm 25 & \pm 0.2 \\
\hline
\end{tabular}

$\varepsilon_{\mathrm{T}}$ : Elongation at break; ${ }^{* *} \varepsilon_{\mathrm{B}}$ : deflection

According to the data, the composite samples containing the treated modified sawdust particles were characterized by an increase in the mechanical strength and stiffness, compared with the recycled polypropylene composites reinforced with the milled initial aspen sawdust. The maximum tensile strength and the tensile modulus of rupture of the composite samples filled with the treated hydrolyzed and treated ammoxidized particles increased an average of $46 \%$ and $52 \%$, respectively, relative to the same indexes of the composite samples filled with the initial sawdust. The maximum bending strength and the bending modulus of the composites incorporating the treated hydrolyzed and treated ammoxidized particles grew 1.4 to 1.5 and 2.1 to 2 .2-fold, respectively. Simultaneously, the deformability of these composites essentially decreased, namely the elongation at break $(\varepsilon T)$ dropped more than 2.3 to 2.4 times, but deflection ability $(\varepsilon \mathrm{B})$ diminished more than 3.2 to 3.6 times, compared with the composite filled with the initial sawdust. A relatively minor improvement in the mechanical properties of the WPC sample containing the treated ammoxidized sawdust in comparison with the sample filled with the treated hydrolyzed sawdust may have been caused by the minor difference in their water wettability.

Scanning electron microscope images of the rPP-based wood-plastic composites are shown in Fig. 6. The SEM surface image of the WPC sample filled with the initial sawdust $(A, C)$ shows the non-uniform surface with the presence of microcracks and other surface defects. On its cross-section, pullouts of the microparticles, debonding, and the presence of internal voids can be observed. This may provide evidence of weak adhesion between the unmodified sawdust microparticles and rPP. At the same time, the surface of the composite sample containing the treated ammoxidized microparticles was 
characterized by homogeneity (B); the particles pullout and debonding as well as the formation of voids (D) on its cross-section are minimal, which may confirm the good interfacial adhesion between the treated modified sawdust microparticles and rPP in the presence of the new adhesion enhancer.
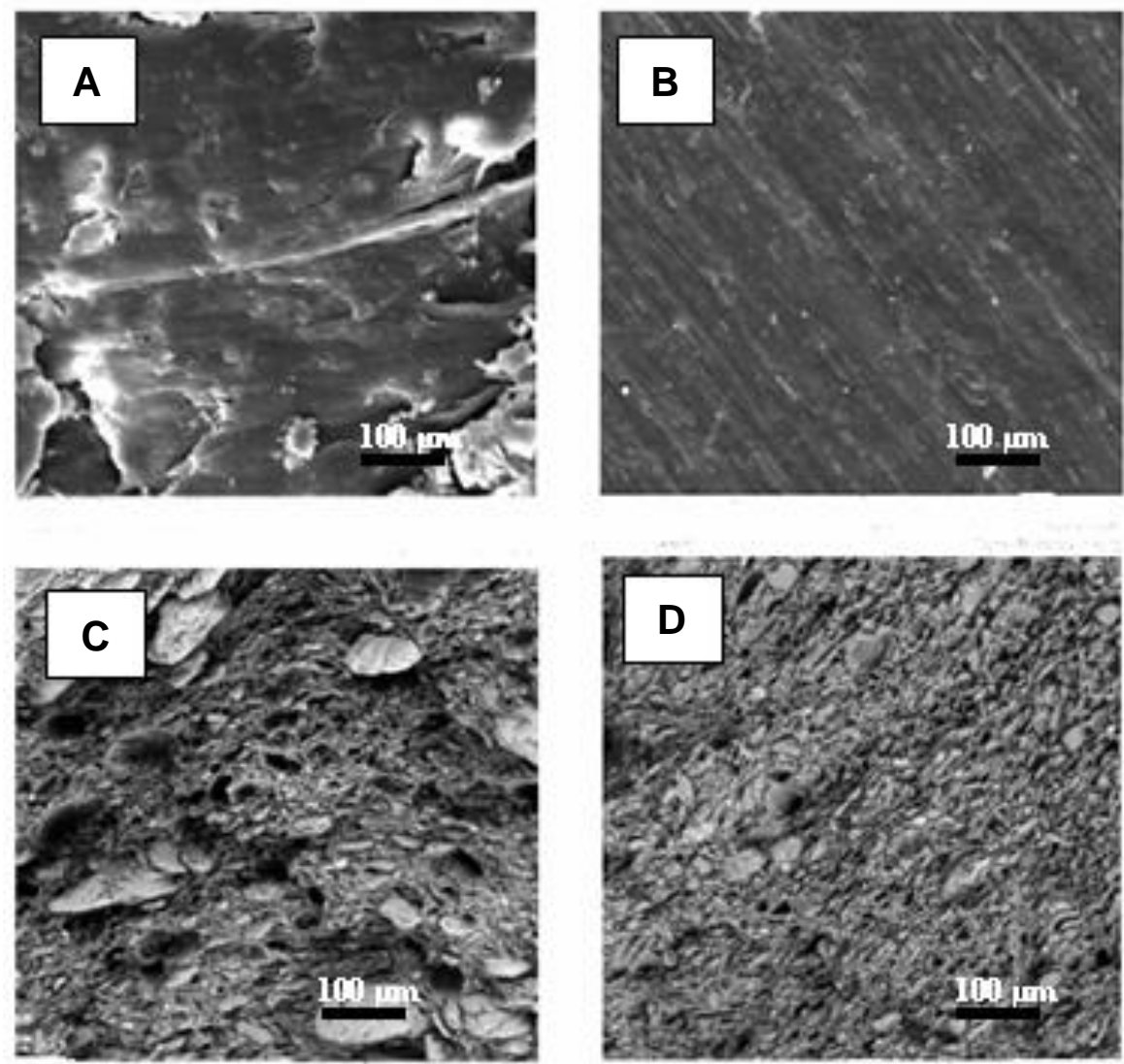

Fig. 6. Images of surface and cross-section of the composite samples (x 500) containing the (A, C) initial and (B, D) treated ammoxidized sawdust microparticles

\section{CONCLUSIONS}

1. The water-soluble, non-stoichiometric polyelectrolyte complex-hydrolyzed aspen soda lignin (HASL)/polyethylenimine (PEI) — formed the nanoparticles in alkaline water solution. Their average diameter was smaller than the size of the lignin nanoparticles, and close in size to the PEI ones. The lignin-containing polyelectrolyte complexes (LPEC) nanoparticles had a lower surface tension at the air-water interface in comparison with the initial components, as well as a lower specific viscosity than the additive value of specific viscosities of the lignin and PEI. The formation of the LPEC nanoparticles was regarded as a result of hydrogen and electrostatic interactions between the components.

2. The treatment with the LPEC nanoparticles led to an increase of the fixed nitrogen content in the modified (hydrolyzed and ammoxidized) aspen sawdust microparticles. This caused a decrease in the amount of adsorbed water, an increase in the contact 
angles values, and a reduction in the polar part of the total surface free energy (SFE) of the modified wood. The decreasing ability to be wetted by water may be a result of the formation of imine and amide bonds between the free amino groups of the adsorbed LPEC nanoparticles and the carbonyl and carboxyl groups of the lignocellulosic matrix of the modified sawdust. The lower contact angle of the ammoxidized sawdust particles, in comparison with the hydrolyzed sawdust ones, may be caused by both the insufficient amount of the formed amide bonds onto the ammoxidized wood surface, due to the low content of the fixed nitrogen, and the presence of the hydrophilic carboxylic groups formed during the ammoxidation.

3. The composite samples containing the modified aspen sawdust, treated with the LPEC nanoparticles, were characterized by the improved mechanical properties and the decreased water's wettability in comparison with the samples filled with the initial sawdust. The enhanced interfacial adhesion was explained by the formation of covalent and physicochemical bonds between the nitrogen-containing groups of the treated sawdust particles and the oxygen-containing groups of rPP during the processing. A relatively minor improvement in the mechanical properties for the wood-plastic composites (WPC) sample filled with the treated ammoxidized sawdust, compared to the composite sample containing the treated hydrolyzed sawdust, was explained by the negligible difference in their hydrophobicity.

\section{ACKNOWLEDGEMENTS}

The authors thank the Ministry of Education and Science of the Republic of Latvia for the financial support of the National Research Programme (ResProd, 2014-2018) and the Biogrant (1/2019).

\section{REFERENCES CITED}

Adamson, A. W., and Gast, A. P. (1997). Physical Chemistry of Surfaces, John Wiley \& Sons, New York, NY, USA.

Ankerfors, C., Lingström, R., Wågberg, L., and Ödberg, L. (2009). “A comparison of polyelectrolyte complexes and multilayers: Their adsorption behavior and use for enhancing tensile strength of paper," Nord. Pulp Pap. Res. J. 24(1), 77-86. DOI: 10.3183/npprj-2009-24-01-p077-086

ASTM D638 (2007). "Standard test method for tensile properties of plastics," ASTM International, West Conshohocken, PA, USA.

Carus, M., Eder, A., Dammer, L., Korte, H., Scholz, L., Essel, R., and Breitmayer, E. (2014). "Wood-plastic composites (WPC) and natural fiber composites (NFC): European and global markets 2012 and future trends in automotive and construction," Bioplastics Magazine 10, 12-13.

De La Orden, M. U., Sánchez, G. C., Quesada, M. G., and Urreaga, J. M. (2007). "Novel polypropylene-cellulose composites using polyethylenimine as coupling agent," Compos. Part A-Appl. S. 38(9), 2005-2012. DOI: 10.1016/j.compositesa.2007.05.008

De La Orden, M. U., Sánchez, C. G., Quesada, M. G., and Urreaga, J. M. (2010). "Effect of different coupling agents on the browning of cellulose-polypropylene composites 
during melt processing," Polym. Degrad. Stabil. 95(2), 201-206. DOI: 10.1016/j.polymdegradstab.2009.11.024

Della Volpe, C., and Siboni, S. (2018). "The Wilhelmy method: A critical and practical review," Surface Innovations 6(3), 120-132. DOI: 10.1680/jsuin.17.00059.

Faix, O. (1992). "Fourier transform infrared spectroscopy," in: Methods in Lignin Chemistry, S. Y. Lin, and C. W. Dence (ed.), Springer, Berlin, Germany, pp. 233-241.

Farsi, M. (2012). "Effect of surface modification on thermo-mechanical behaviour of wood-polymer composite," Asian J. Chem. 24(6), 2775-2779.

Faruk, O., Bledzki, A. K., Fink, H.-P., and Sain, M. (2012). "Biocomposites reinforced with natural fibers: 2000-2010," Prog. Polym. Sci. 37(11), 1552-1596. DOI: 10.1016/j.progpolymsci.2012.04.003

Gärdlund, L., Norgren, M., Wågberg L., and Marklund, A. (2007). "The use of polyelectrolyte complexes (PEC) as strength additives for different pulps used for production of fine paper," Nord. Pulp Pap. Res. J. 22(2), 210-216. DOI: 10.3183/NPPRJ-2007-22-02-p210-216

Gärdlund, L., Wågberg, L., and Gernandt, R. (2003). "Polyelectrolyte complex for surface modification of wood fibres. II. Influence of complexes on wet and dry strength of paper," Colloids Surf. A, Physicochem. Eng. Asp. 218(1-3), 137-149. DOI: 10.1016/S0927-7757(02)00588-5

Hu, L., Stevanovic, T., and Rodrigue, D. (2014). "Lignin-based thermoplastic composites and compatibilization methods," in: Structural Analysis, Applications in Biomaterials and Ecological Significance, F. Lu (ed.), NovaScience Publishers, New York, NY, USA, pp. 253-282.

Hubbe, M. A., Rojas, O. J., Lucia, L. A., and Sain, M. (2008). “Cellulosic nanocomposites: A review," BioResources 3(3), 929-980. DOI: 10.15376/biores.3.3.929-980

Hubbe, M. A. (2006). "Bonding between cellulosic fibers in the absence and presence of dry-strength agents - A review," BioResources 1(2), 281-318. DOI: 10.15376/biores.1.2.281-318

ISO 178 (2010). "Plastics. Determination of flexural properties," International Organization for Standardization, Geneva, Switzerland.

Kabanov, V. A. (2005). "Polyelectrolyte complexes in solution and in bulk," Russ. Chem. Rev. 74(1), 3-20.

Kabir, M. M., Wang, H., Lau, K. T., and Cardona, F. (2012). "Chemical treatments on plant-based natural fiber reinforced polymer composites: An overview," Compos. Part B-Eng. 43(7), 2883-2892. DOI: 10.1016/j.compositesb.2012.04.053

Karunarathna, M., and Smith R.C. (2020). "Valorization of lignin as a sustainable component of structural materials and composites: Advances from 2011 to 2019," Sustainability 12(2), Article number 734. DOI: 10.3390/su12020734

Korhonen, J., Honkasalo, A., and Seppälä, J. (2018). "Circular economy: The concept and its limitations," Ecol. Econ. 143, 37-46. DOI: 10.1016/j.ecolecon.2017.06.041

Kun, K., and Pukanszky, B. (2017). "Polymer/lignin blends: Interactions, properties, applications,” Eur. Polym. J. 93, 618-641. DOI: 10.1016/j.eurpolymj.2017.04.035

La Mantia, F. P., and Morreale, M. (2011). "Green composites: A brief review," Compos. Part A-Appl. S. 42(6), 579-588. DOI: 10.1016/j.compositesa.2011.01.017 
Lankalapalli, S., and Kolapalli, V. R. M. (2009). "Polyelectrolyte complexes: A review of their applicability in drug delivery technology," Indian J. Pharm. Sci. 71(5), 481-487. DOI: $10.4103 / 0250-474 X .58165$

Lappan, R., Pelton, R., McLennan, I., Patry, J., and Hrymak, A. (1997). "Kraft lignin poly(DADMAC) precipitate formation,” Ind. Eng. Chem. Res. 36(4), 1171-1175.

DOI: 10.1021/ie960460u

Li, P., and Pelton, R. (1992). "Wood pulp washing 1. Complex formation between kraft lignin and cationic polymers," Colloid. Surface. 64(3-4), 217-222. DOI: 10.1016/0166-6622(92)80102-8

Lu, J. Z., Wu, Q., and McNabb, H. S. (2000). "Chemical coupling in wood fiber and polymer composites: A review of coupling agents and treatments," Wood Fiber. Sci. 32(1), 88-104.

Mariotti, N., Srevanovic, T., Rodrigue, D., and Wang, X. (2014). "Esterified kraft lignin: A potential coupling agent for wood plastic composites (WPC)," in: Lignin: Structural Analysis, Applications in Biomaterials and Ecological Significance, F. Lu (ed.), Nova Science Publishers, New York, NY, USA, pp. 283-308.

Maximova, N., Laine, J., and Stenius, P. (2005). "Adsorption of lignin-cationic starch complexes on cellulose fibres and their effect on sheet properties," Paperi Ja Puu 87(3), 176-182.

Najafi, S. K. (2013). "Use of recycled plastics in wood plastic composites - A review," Waste Manage. 33(9), 1898-1905. DOI: 10.1016/j.wasman.2013.05.017

Pergushov, D. V., Borisov, O. V., Zezin, A. B., and Müller, A. H. E. (2010). "Interpolyelectrolyte complexes based on polyionic species of branched topology," in: Self Organized Nanostructures of Amphiphilic Block Copolymers, A. H. E. Müller and O. Borisov (eds.), Springer, Berlin, Heidelberg, Germany, pp. 131-161. DOI: 10.1007/12_2010_102

Pickering, K. L., Efendy, M. A., and Le, T. M. (2016). "A review of recent developments in natural fibre composites and their mechanical performance," Compos. Part AAppl. S. 83, 98-112. DOI: 10.1016/j.compoositesa.2015.08.038

Sarkanen, K. V., and Ludwig, C. H. (1971). Lignins: Occurrence, Formation, Structure and Reactions, John Wiley \& Sons, New York, NY, USA.

Shulga, G. M., Kalyuzhnaya, R. I., Mozheyko, L. N., Rekner, F. V., Zezin, A. B., and Kabanov, V. A. (1984). "Features of the reactions between lignosulphonates and polymeric bases in aqueous solutions," Polym. Sci. 26(2), 319-324. DOI: 10.1016/0032-3950(84)90110-2

Shulga, G. M., Mozeyko, L. N., Rekner, F. V., Kalyuzhmajya, R. I., Zezin, A. B., and Kabanov, V. A. (1982). "Chemical conversions in polyelectrolyte complexes based on lignosulfonates," Khim. Drev. [Chemistry of Wood] 1, 87-93.

Shulga, G., Rekners, F., and Varslavans, J. (2001). "SW-soil and water: Lignin-based interpolymer complexes as a novel adhesive for protection against erosion of sand soil," J. Agr. Res. 78(3), 309-316. DOI: 10.1006/jaer.2000.0599

Shulga, G., Kalyuzhnaya, R., Zezin, A., and Kabanov, V. (2002). "Effect of the molecular mass of lignosulphonate on the interaction with polymeric cation in dilute aqueous solutions and the properties of products formed," Cell. Chem. Technol. $36(4), 225-241$. 
Shulga, G., Shakels, V., Aniskevicha, O., Bikova, T., and Treimanis, A. (2006). "Effect of alkaline modification on viscometric and surface-active properties of soluble lignin," Cell. Chem. Technol. 40(6), 383-392.

Shulga, G., Betkers, T., Shakels, V., Neiberte, B., Verovkins, A., Brovkina, J., Belous, O., Ambrazaitene, D., and Žukauskaite, A. (2007). "Effect of the modification of lignocellulosic materials with a lignin-polymer complex on their mulching properties," BioResources 2(4), 572-582.

Shulga, G., Shakels, V., Aniskevicha, O., Zakharova, J., and Skudra, S. (2009). "Interfacial properties of polyelectrolyte complexes incorporating kraft lignin," Holzforschung 63(6), 711-714. DOI: 10.1515/HF.2009.126

Shulga, G., Shakels, V., Dzenis, M., Livcha, S., and Jaunslavietis, J. (2016a). "Ligninchitosan polyelectrolyte complex as a biomacrosurfactant," in: Proceedings of the $14^{\text {th }}$ European Workshop on Lignocellulosics and Pulp (EWLP), C. Chirat, N. Marlin, and C. Boisset-Helbert (eds.), EWLP Committee, Autrans, France, pp. 225-228.

Shulga, G., Neiberte, B., Verovkins, A., Vitolina, S., Jaunslavietis, J., and Ozolins, J. (2016b). "Short fibre filler from wood residue for polymeric composite materials," Mater. Sci. 22(3), 370-375. DOI: 10.5755/j01.ms.22.3.8752

Shulga, G., Neiberte, B., Verovkins, A., Jaunslavietis, J., Shakels, V., Vitolina, S., and Sedliačik, J. (2016c). "Eco-friendly constituents for making wood-polymer composites," Key Eng. Mater. 688, 122-130. DOI:

10.4028/www.scientific.net/KEM.688.122

Ström, G., and Stenius, P. (1981). "Formation of complexes, colloids and precipitates in aqueous mixtures of lignin sulphonate and some cationic polymers," Colloid. Surface. 2(4), 357-371. DOI: 10.1016/0166-6622(81)80022-4

Taib, R. M., Ishak, Z. A. M., Rozman, H. D., and Glasser, W. G. (2006). "Effect of moisture absorption on the tensile properties of steam-exploded Acacia mangium fiber-polypropylene composites," J. Thermoplast. Compos. Mater. 19(5), 475-489. DOI: $10.1177 / 0892705706062208$

TAPPI T203 cm-99 (1999). “Alpha-, beta- and gamma-cellulose in pulp,” TAPPI Press, Atlanta, GA, USA.

TAPPI T222 om-02 (2002). “Acid-insoluble lignin in wood and pulp,” TAPPI Press, Atlanta, GA, USA.

TAPPI T264 om-97 (1997). "Preparation of wood for chemical analysis," TAPPI Press, Atlanta, GA, USA.

Tsuchida, E. (2008). "Formation of polyelectrolyte complexes and their structures," $J$. Macromol. Sci. A. 31(1), 1-15. DOI: 10.1080/10601329409349713

Turku, I., Kärki, T., and Puurtinen, A. (2018). "Durability of wood plastic composites manufactured from recycled plastic," Heliyon 4(3), e00559. DOI: 10.1016/j.heliyon.2018.e00559

Verma, A., and Verma, A. (2013). "Polyelectrolyte complex-an overview," Int. J. Pharm. Sci. Res. 4(5), 1684-1691. DOI: 10.13040/IJPSR.0975-8232.4(5).1684-91

Wågberg, L., and Annergren, G. (1997). "Physicochemical characterization of papermaking fibres," in: The Fundamentals of Papermaking Materials, C. F. Baker (ed.), Pira International, Leatherhead, Surrey, UK, pp. 1-82.

Yuan, Y., Guo, M. H., and Liu, F. Y. (2014). "Preparation and evaluation of green composites using modified ammonium lignosulfonate and polyethylenimine as a binder," BioResources 9(1), 836-848. 
Zakis, G. (1994). Functional Analyses of Lignin and Their Derivatives, TAPPI Press, Atlanta, GA, USA.

Article submitted: July 12, 2020; Peer review completed: November 27, 2020; Revised version received and accepted: February 18, 2021; Published: February 25, 2021.

DOI: 10.15376/biores.16.2.2804-2823 